\title{
Serum Interleukin-15 Levels Are Associated with Severity of Pain in Patients with Knee Osteoarthritis
}

\author{
Jian-Min Sun, ${ }^{1}$ Liang-Zhi Sun, ${ }^{1}$ Jun Liu, ${ }^{2}$ Bao-hui Su, ${ }^{1}$ and Lin Shi ${ }^{1}$ \\ ${ }^{1}$ Department of Hand-Foot Surgery, Weifang People's Hospital, Weifang 261041, Shandong, China \\ ${ }^{2}$ Department of Orthopedic Trauma, Weifang People's Hospital, Weifang 261041, Shandong, China
}

Correspondence should be addressed to Liang-Zhi Sun; wfsunlz@126.com

Received 2 April 2013; Accepted 12 July 2013

Academic Editor: Paul Ashwood

Copyright (C) 2013 Jian-Min Sun et al. This is an open access article distributed under the Creative Commons Attribution License, which permits unrestricted use, distribution, and reproduction in any medium, provided the original work is properly cited.

Background. Inflammation plays a crucial role in the development and progression of osteoarthritis (OA). Interleukin-15 (IL-15) is a well-known proinflammatory cytokine. Objective. We aimed at evaluating the relationship between serum IL-15 levels and the severity of pain as well as radiographic progression in patients with knee OA. Methods. Two hundred and twenty-six OA patients and 106 controls were enrolled in this study. The symptomatic/radiological severity of OA was assessed by the Western Ontario McMaster University Osteoarthritis Index- (WOMAC-)pain scores/Kellgren-Lawrence (KL) grading system. Serum IL-15 levels were measured by enzyme-linked immunosorbent assay (ELISA). Results. Serum IL-15 levels were significantly higher in OA patients compared with controls. Serum IL-15 levels were independently and positively correlated with WOMAC-pain scores but not KL grades in OA patients. Conclusions. We demonstrated that increased serum IL-15 levels were independently correlated with self-reported greater pain in knee OA patients. These results suggest that IL-15 might play a crucial role in the pathogenesis of OA related pain and therapeutic interventions by blocking IL-15 signaling pathways to delay the degenerative process of OA related pain which warrants further investigations.

\section{Introduction}

Osteoarthritis (OA) is the most widespread joint-affecting disease characterized by articular cartilage degeneration, subchondral sclerosis, osteophyte formation, and synovial inflammation [1]. As a part of the ageing process, OA is the most common arthritis of the elderly and may lead to severe symptoms like pain, malformation of the joint, and disability [2]. All joints may be affected, but the knee is the most clinically significant site of primary OA involvement [3].

Since the pain sensation is the primary complaint that leads to physical functional limitations and reduced quality of life in patients with knee OA, there has been a long-standing need for identification of reliable biomarkers in order to facilitate the design of proper therapies directed at underlying mechanisms of knee pain. Although the exact mechanism of knee pain in OA disease is unclear, it is now well appreciated that inflammatory molecules, such as proinflammatory cytokines, are among the critical mediators of the disturbed processes implicated in pain-related pathophysiology [4]. Therefore, inflammatory molecules hold the potential to be assessed as pain-related biomarkers owing to the inflammatory nature of the disease.

Interleukin-15 (IL-15), initially described as a $\mathrm{T}$ cell growth factor, is a $14-15 \mathrm{kDa}$ innate response cytokine that regulates $\mathrm{T}$ and natural killer (NK) cell activation and proliferation [5]. As a proinflammatory cytokine, IL-15 is believed to be implicated in pathophysiology of arthritis through multiple mechanisms, especially for patients with rheumatoid arthritis (RA) [6]. Blockade of IL-15 has been met with therapeutic success in RA patients [7]. However, the relationship between circulating IL-15 levels and the severity of knee pain in OA patients has not been assessed until now. Additionally, a previous study demonstrated an association between radiographic features of knee OA and pain [8]. Therefore, the present study is aimed at evaluating the relationship between circulating IL-15 levels and the severity of pain as well as radiographic progression in patients with knee OA. 


\section{Materials and Methods}

2.1. Study Subjects. From April 2010 to November 2012, 226 patients with primary knee OA who visited our hospital were recruited. Knee OA was diagnosed according to the American College of Rheumatology revised criteria for OA. One hundred and six age and sex matched volunteers who visited our hospital for routine physical examination were enrolled as controls. Participants were excluded on the base of having RA, posttraumatic arthritis, previous joint infection, crystal deposition arthritis, enteropathic arthritis, hemophilic arthropathy, systemic inflammatory or autoimmune disorders, malignant disease, advanced renal disease, and histories of corticosteroids medication. Written informed consent was obtained from all subjects, and the study protocol was approved by the ethics committee of our hospital.

2.2. Pain Evaluation. The pain severity of each patient was evaluated according to the Western Ontario McMaster University Osteoarthritis Index- (WOMAC-) pain scores. The WOMAC-pain score consists of 5 questions that assess a patient's level of pain as it is related to functional activities [9]. These activities include walking on a flat surface, going up or down stairs, at night while in bed, sitting or lying, and standing upright. The responses were scored with the use of a $0-4$ rating scale, where 0 represents no pain and a higher score represents greater pain. The total WOMAC-pain score is the sum of each response score. The total range of possible WOMAC-pain score is from 0 to 20 . This scale is very widely used, and the reliability and validity have been well established in OA population [9].

2.3. Radiographic Grading of $O A$. The anteroposterior radiographs of the affected joints were obtained to evaluate radiographic severity of OA using the Kellgren and Lawrence (KL) classification as follows: grade 1 , questionable narrowing of joint space and possible osteophytic lipping; grade 2, definite osteophytes and possible narrowing of joint space; grade 3, moderate multiple osteophytes, definite narrowing of joints space, some sclerosis, and possible deformity of bone contour; grade 4, large osteophytes, marked narrowing of joint space, severe sclerosis, and definite deformity of bone contour [10].

2.4. Laboratory Examinations. Blood samples were obtained from the antecubital vein of all participants in the fasting state at 7:00 to 7:30 AM. After clotting, blood samples were centrifuged and stored at $-80^{\circ} \mathrm{C}$ until examination. Serum IL-15 levels were measured using commercially available sandwich enzyme-linked immunosorbent assay (ELISA) kits (R\&D Systems, CA, USA) according to the manufacturer's protocol. The intra-assay CV and interassay CV of the ELISA kits were $<5 \%$ and $<10 \%$, respectively. The recovery of spiked standard in serum was $98 \%$. All the samples were routinely analyzed by ELISA in triplicate, and the results were averaged.

2.5. Statistical Analysis. The normality of continuous variables was assessed using the Kolmogorov-Smirnov test. Continuous data were expressed as mean value \pm SD or median (interquartile range), while categorical data are presented as $n$ (percentages). Differences between the two groups were analyzed using unpaired $t$-test, Mann-Whitney $U$ test, or Chi-square test as indicated. Differences among groups were analyzed by one-way analysis of variance (ANOVA) followed by Tukey post hoc analysis, Kruskal-Wallis analysis, or Chisquare test when appropriate. Spearman coefficients were performed to assess the correlation between serum IL-15 levels and KL grades/WOMAC-pain scores. Multivariate linear regression modeling was used to evaluate the independent predictors of WOMAC-pain scores. Statistical analyses were performed by using the Statistical Package for Social Sciences (SPSS 16.0 for windows; SPSS Inc., Chicago, IL, USA). Differences were considered statistically significant at a $P$ value of $<0.05$.

\section{Results}

3.1. Baseline Clinical Characteristics. The baseline clinical data of the controls and OA patients were presented in Table 1. The controls and OA patients were similar with regard to age, proportion of sexes, and body mass index (BMI) $(P>0.05)$. There was also no significant difference in baseline clinical characteristics across categories of OA radiographic severity (based on KL classification) $(P>0.05)$.

3.2. Serum IL-15 Levels. As shown in Table 1, OA patients had significantly elevated serum IL-15 levels when compared to controls (6.04 (range 4.82 to 8.96$) \mathrm{pg} / \mathrm{mL}$ versus 2.17 (range $1.53-3.37) \mathrm{pg} / \mathrm{mL}, P<0.01$ ). In OA patients, serum IL15 levels were analyzed according to the KL classification. Kruskal-Wallis analysis demonstrated that serum IL-15 levels tended to be lower with the increment of KL grade, though the difference did not reach statistically significant (Table 1).

\subsection{Correlation of Serum IL-15 Levels with WOMAC-Pain} Scores and KL Grades. The median (interquartile range) of WOMAC-pain scores in OA patients is 11 (range 8 to 18), which is in accordance with the range of WOMAC-pain scores in other Chinese OA populations reported in the literature [11]. We showed that serum IL-15 levels were positively correlated with WOMAC-pain scores in OA patients $(r=$ $0.279, P<0.001$; Figure 1). Multivariate linear regression analysis revealed that the correlation between serum IL15 levels and WOMAC-pain scores was independent after controlling the influence of age, gender, and BMI $(\beta: 0.339$, $t=5.283$, and $P<0.001$ ). However, serum IL-15 levels were not significantly associated with KL grades in OA patients $(r=-0.116, P=0.081)$. In addition, there was also no significant correlation between WOMAC-pain scores and KL grades in OA patients $(r=0.115, P=0.085)$.

\section{Discussion}

The present study examined the relationship between serum IL-15 levels and pain scale scores and radiographic severity in patients with knee OA. We demonstrated for the first time that serum IL-15 levels were independently associated with 
TABLE 1: Clinical characteristics and serum IL-15 levels.

\begin{tabular}{lccccc}
\hline & $\begin{array}{c}\text { Controls } \\
(n=106)\end{array}$ & $\begin{array}{c}\text { OA patients } \\
\text { Total }(n=226)\end{array}$ & $\begin{array}{c}\text { KL grade } 2 \\
(n=99)\end{array}$ & $\begin{array}{c}\text { KL grade 3 } \\
(n=74)\end{array}$ & $\begin{array}{c}\text { KL grade } 4 \\
(n=53)\end{array}$ \\
\hline Age $($ years $)$ & $63.61 \pm 11.15$ & $64.01 \pm 9.49$ & $64.64 \pm 10.31$ & $63.86 \pm 8.53$ & $63.06 \pm 9.27$ \\
Female $(n, \%)$ & $61,57.55 \%$ & $139,61.50 \%$ & $64,64.65 \%$ & $45,60.81 \%$ & $30,56.60 \%$ \\
BMI $\left(\mathrm{Kg} / \mathrm{mm}^{2}\right)$ & $22.43 \pm 2.28$ & $23.10 \pm 2.04$ & $23.31 \pm 2.24$ & $22.94 \pm 1.78$ & $22.96 \pm 1.99$ \\
IL-15 $(\mathrm{pg} / \mathrm{mL})$ & $2.17(1.53-3.37)$ & $6.04(4.82-8.96)$ & $6.35(5.09-10.09)$ & $6.00(4.35-8.47)$ & $5.85(4.10-7.38)$ \\
WOMAC-pain scores & & $11(8-18)$ & $10(9-14)$ & $11(6-18)$ & $14(9-18)$ \\
\hline
\end{tabular}

All values are expressed as the mean value \pm SD or $n$ (\%). OA: osteoarthritis; KL: Kellgren-Lawrence; BMI: body mass index; IL-15: interleukin-15. ${ }^{*} P<0.01$ compared with controls.

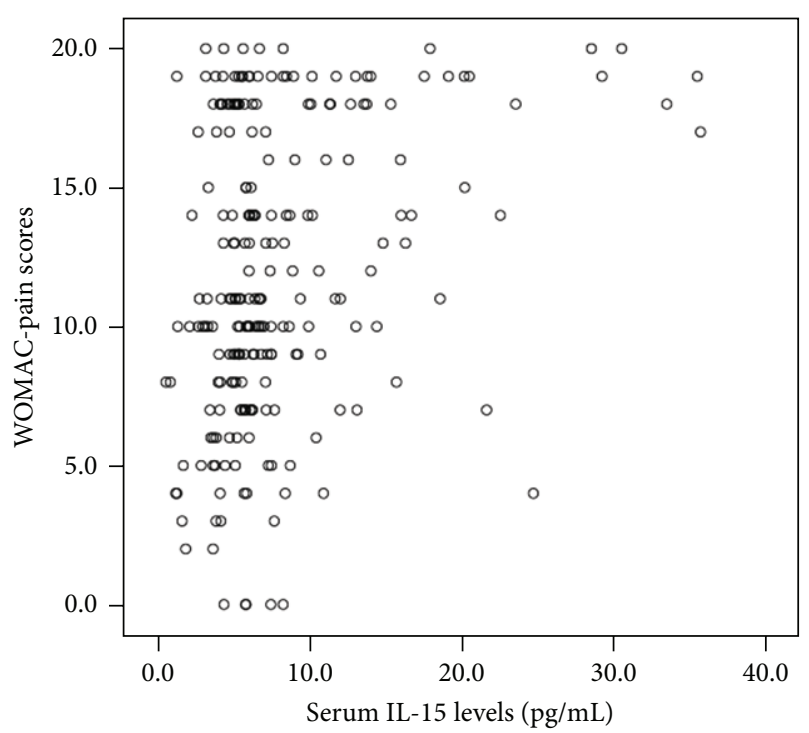

FIgURE 1: Correlations of serum IL-15 levels with WOMAC-pain scores, WOMAC: Western Ontario and McMaster Universities Osteoarthritis Index.

the intensity of pain but not radiographic severity in OA patients. These results indicated that IL-15 may represent a new potential serum biochemical marker for reflecting the severity of pain in OA patients.

The identification of novel OA biomarkers could provide useful diagnostic information by reflecting disease-relevant biological activity and predicting the course of disease progression. Besides, reliable biomarkers could assist in identifying potential targets for therapeutic interventions and thus facilitate therapeutic decision making. Therefore, the insights provided by OA related biomarker studies represent a paradigm shift in the methods of diagnosis and treatment of OA. There is growing evidence that systemic markers of inflammation are associated with severity or clinical course of OA [4]. Therefore, proinflammatory cytokines are being explored as biomarkers for early OA diagnosis and for monitoring disease progression.

We demonstrated in this study that serum IL-15 levels were positively associated with WOMAC-pain scores in OA patients. This association remained significant after the adjustment of potential confounders such as age, gender, and BMI, indicating that OA patients with high serum IL-15 levels may suffer a more severe self-reported pain. Increasing evidence indicated that chronic inflammation played an important pathophysiological role in the generation and maintenance of pain in OA patients [12]. A previous study by Penninx et al. demonstrated that high concentrations of inflammatory markers in the serum were associated with increased pain of OA [13]. IL-15 is a potent proinflammatory cytokine that plays a major role in the development of inflammatory and protective immune responses by modulating immune cells of both the innate and adaptive immune systems [14]. IL-15 promotes the production of inflammatory cytokines such as tumor necrosis factor- $\alpha$ (TNF- $\alpha$ ) and interleukin- (IL-) 6 in the synovial membrane via effects on synovial $\mathrm{T}$ cells $[7,15]$. These inflammatory cytokines have been reported to contribute to OA pain by increasing cartilage degradation and inducing hyperalgesia via a number of direct and indirect actions [16]. Therefore, the proinflammatory role of IL-15 might explain the relationship between serum IL-15 levels and the severity of OA pain observed in our study. However, the correlation between serum IL-15 levels and WOMAC-pain scores was weak, and its clinical significance is yet to be further investigated.

There is a general opinion that an association exists between radiographic features of $\mathrm{OA}$ and knee pain [8]. However, many researchers believed that the association is modest $[17,18]$. Many patients with advanced radiographic $\mathrm{OA}$ have no pain and a fair proportion of patients with severe knee pain show no radiographic features of OA [19]. We also investigated the relationship between serum IL15 levels and radiographic features of $\mathrm{OA}$ in this study. We found that serum IL-15 levels were not significantly correlated with radiographic grading of OA. In addition, serum IL-15 levels seemed to be lower with the increasing radiographic severity of knee OA, though the difference was not statistically significant. These results were in accordance with two recent studies. Ling et al. suggested that serum IL15 levels were associated with both established disease and new development of knee and hand OA [20]. Scanzello et al. further demonstrated that IL-15 concentrations in synovial fluid (SF) of early knee OA patients were significantly elevated when compared to end-stage OA patients [21]. All these results revealed that the pathophysiological role of IL-15 in promoting progressive cartilage loss might be more active in early stage of OA. However, further studies are needed to clearly delineate molecular pathways which IL-15 activation may have in the pathophysiology of OA. 
The limitations of this study should be taken into account when interpreting our results. Firstly, this study was crosssectional with a relatively small number of Chinese patients. Therefore, further prospective longitudinal studies with a larger population and a wider range of race are needed to verify our findings. Secondly, we only measured IL-15 levels in serum samples of OA patients; the detection of IL-15 expression in SF and synovial membrane of OA patients might reveal more valuable information on the potential pathophysiological role of IL-15. Thirdly, OA knee pain includes many types of pain, such as night pain, rest pain, and activity-related pain. Our study focused only on a 0-4 rating pain scale in a limited number of patients during a short period. Fourthly, only serum IL-15 levels were examined in our study. Additional studies on the investigation of other inflammatory biomarkers (e.g., C-reactive protein and IL1) can provide more valuable information on the diseasepromoting role of IL-15 signaling pathways in OA related pain and to truly appreciate IL-15 uniqueness.

To summarize, the present study demonstrated that increased serum IL-15 levels were independently correlated with self-reported greater pain in knee OA patients. The results suggest that IL-15 might play a crucial role in the pathogenesis of OA related pain and therapeutic interventions by blocking IL-15 signaling pathways to delay the degenerative process of OA related pain which warrants further investigations.

\section{Conflict of Interests}

The authors declare that they have no conflict of interest.

\section{References}

[1] L. Pulsatelli, O. Addimanda, V. Brusi, B. Pavloska, and R. Meliconi, "New findings in osteoarthritis pathogenesis: therapeutic implications," Therapeutic Advances in Chronic Disease, vol. 4, pp. 23-43, 2013.

[2] D. T. Felson, Y. Zhang, M. T. Hannan et al., "The incidence and natural history of knee osteoarthritis in the elderly: the Framingham osteoarthritis study," Arthritis and Rheumatism, vol. 38, no. 10, pp. 1500-1505, 1995.

[3] L. Xu, G. B. Zhu, L. Wang, D. F. Wang, and X. R. Jiang, "Synovial fluid omentin-1 levels are inversely correlated with radiographic severity of knee osteoarthritis," Journal of Investigative Medicine, vol. 60, pp. 583-586, 2012.

[4] M. Kapoor, J. Martel-Pelletier, D. Lajeunesse, J.-P. Pelletier, and H. Fahmi, "Role of proinflammatory cytokines in the pathophysiology of osteoarthritis," Nature Reviews Rheumatology, vol. 7, no. 1, pp. 33-42, 2011.

[5] S. Dubois, J. Mariner, T. A. Waldmann, and Y. Tagaya, "IL$15 \mathrm{R} \alpha$ recycles and presents IL-15 in trans to neighboring cells," Immunity, vol. 17, no. 5, pp. 537-547, 2002.

[6] H. P. Carroll, V. Paunović, and M. Gadina, "Signalling, inflammation and arthritis: crossed signals: the role of interleukin-15 and -18 in autoimmunity," Rheumatology, vol. 47, no. 9, pp. 1269$1277,2008$.

[7] B. Baslund, N. Tvede, B. Danneskiold-Samsoe et al., "Targeting interleukin-15 in patients with rheumatoid arthritis: a proof-of-concept study," Arthritis and Rheumatism, vol. 52, no. 9, pp. 2686-2692, 2005.

[8] T. Neogi, D. Felson, J. Niu et al., "Association between radiographic features of knee osteoarthritis and pain: results from two cohort studies," British Medical Journal, vol. 339, no. 7719, Article ID b2844, pp. 498-501, 2009.

[9] N. Bellamy, W. W. Buchanan, C. H. Goldsmith, J. Campbell, and L. W. Stitt, "Validation study of WOMAC: a health status instrument for measuring clinically important patient relevant outcomes to antirheumatic drug therapy in patients with osteoarthritis of the hip or knee," Journal of Rheumatology, vol. 15, no. 12, pp. 1833-1840, 1988.

[10] J. H. Kellgren and J. S. Lawrence, "Radiological assessment of osteo-arthrosis," Annals of the rheumatic diseases, vol. 16, no. 4, pp. 494-502, 1957.

[11] Z. G. Li, D. W. Zhao, C. J. Xia et al., "Decreased synovial fluid omentin-1 concentrations reflect symptomatic severity in patients with knee osteoarthritis," Scandinavian Journal of Clinical \& Laboratory Investigation, vol. 72, pp. 623-628, 2012.

[12] T. Stürmer, H. Brenner, W. Koenig, and K.-P. Günther, "Severity and extent of osteoarthritis and low grade systemic inflammation as assessed by high sensitivity C reactive protein," Annals of the Rheumatic Diseases, vol. 63, no. 2, pp. 200-205, 2004.

[13] B. W. J. H. Penninx, H. Abbas, W. Ambrosius et al., "Inflammatory markers and physical function among older adults with knee osteoarthritis," Journal of Rheumatology, vol. 31, no. 10, pp. 2027-2031, 2004.

[14] P.-Y. Perera, J. H. Lichy, T. A. Waldmann, and L. P. Perera, “The role of interleukin-15 in inflammation and immune responses to infection: implications for its therapeutic use," Microbes and Infection, vol. 14, no. 3, pp. 247-261, 2012.

[15] I. B. Mcinnes, J. Al-Mughales, M. Field et al., "The role of interleukin-15 in T-cell migration and activation in rheumatoid arthritis," Nature Medicine, vol. 2, no. 2, pp. 175-182, 1996.

[16] S. Orita, T. Koshi, T. Mitsuka et al., "Associations between proinflammatory cytokines in the synovial fluid and radiographic grading and pain-related scores in 47 consecutive patients with osteoarthritis of the knee," BMC Musculoskeletal Disorders, vol. 12, article 144, 2011.

[17] P. A. Dieppe, "Relationship between symptoms and structural change in osteoarthritis: what are the important targets for therapy?" Journal of Rheumatology, vol. 32, no. 6, pp. 1147-1149, 2005.

[18] N. M. Hadler, "Knee pain is the malady-not osteoarthritis," Annals of Internal Medicine, vol. 116, no. 7, pp. 598-599, 1992.

[19] P. Creamer and M. C. Hochberg, "Why does osteoarthritis of the knee hurt-Sometimes?" British Journal of Rheumatology, vol. 36, no. 7, pp. 726-728, 1997.

[20] S. M. Ling, D. D. Patel, P. Garnero et al., "Serum protein signatures detect early radiographic osteoarthritis," Osteoarthritis and Cartilage, vol. 17, no. 1, pp. 43-48, 2009.

[21] C. R. Scanzello, E. Umoh, F. Pessler et al., "Local cytokine profiles in knee osteoarthritis: elevated synovial fluid interleukin-15 differentiates early from end-stage disease," Osteoarthritis and Cartilage, vol. 17, no. 8, pp. 1040-1048, 2009. 


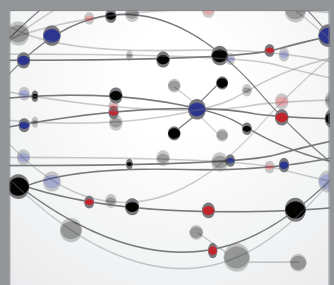

The Scientific World Journal
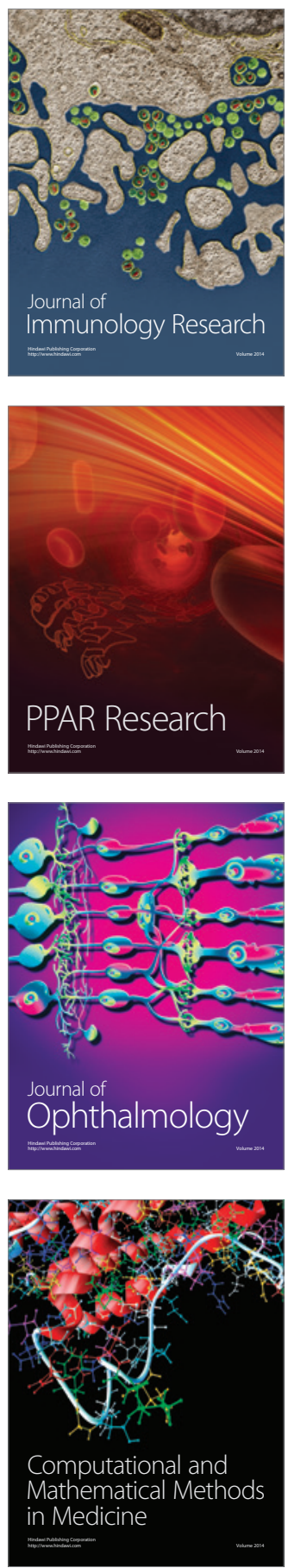

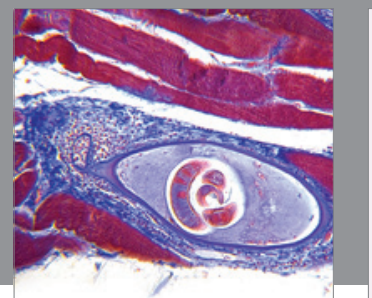

Gastroenterology

Research and Practice
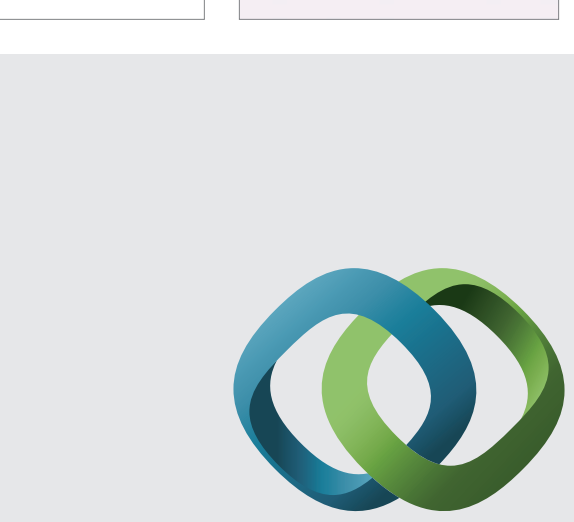

\section{Hindawi}

Submit your manuscripts at

http://www.hindawi.com
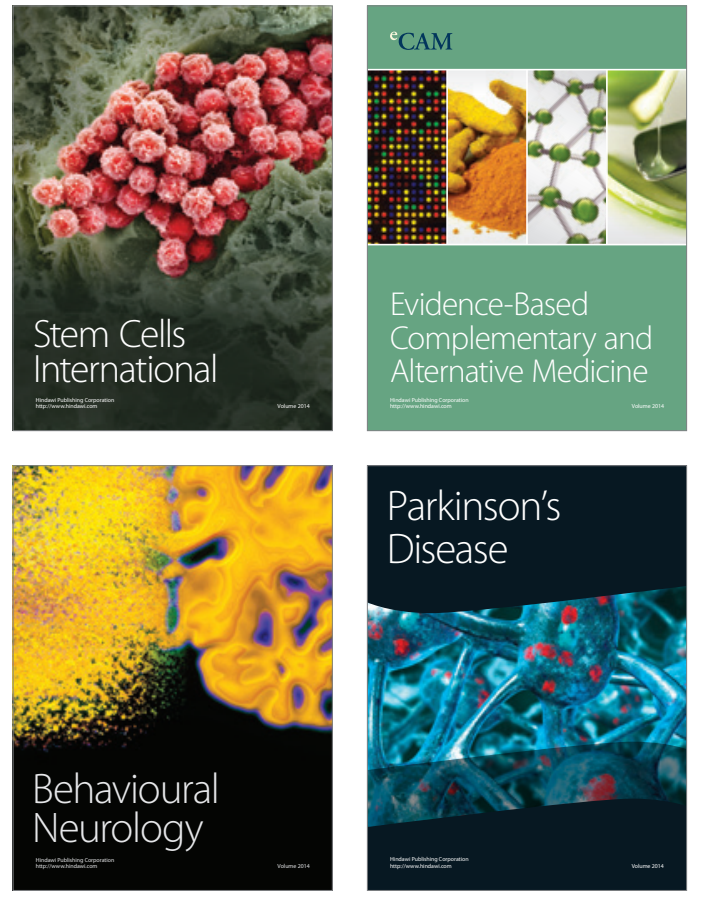
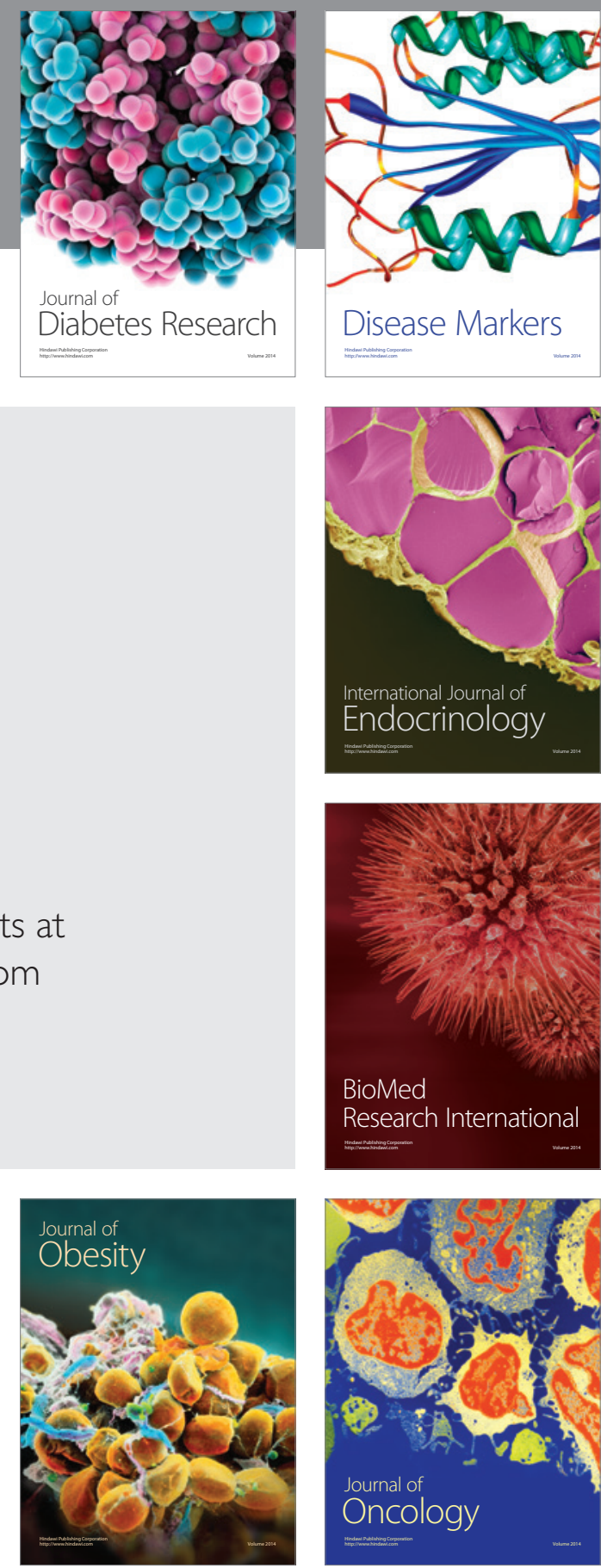

Disease Markers
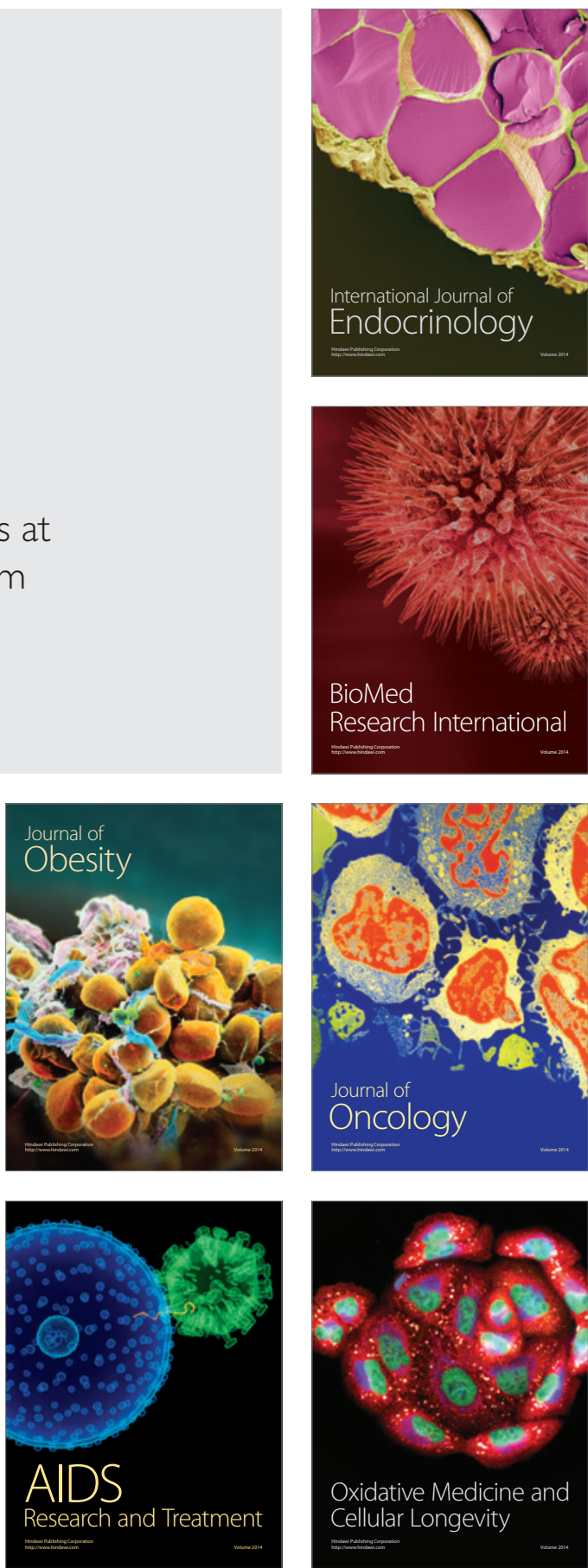